\title{
A Retrospective Cohort Study of the Effect of Hospitalist-Directed Transfers on Patient Flow
}

Yihan Chen, M.D. ' , Inderpreet Saini, M.D. ' , Shivani K. Patel, M.D'2, Holly Wilhalme, M.S. ${ }^{3}$,

William D. Miller, M.D. ${ }^{4}$, and Roswell Quinn, M.D., Ph. D ${ }^{1,5}$

'Department of Medicine, David Geffen School of Medicine, University of California, Los Angeles, CA, USA; ${ }^{2}$ Department of Medicine, Cedar-Sinai Medical Center, Los Angeles, CA, USA; ' ${ }^{3}$ Department of Medicine Statistics Core, University of California Los Angeles, Los Angeles, CA, USA;

${ }^{4}$ Department of Medicine, Section of Pulmonary and Critical Care, University of Chicago, Chicago, IL, USA; ${ }^{5}$ Hospitalist Division of the Department of Medicine, Hospitalist Service, VA Greater Los Angeles Healthcare System, Los Angeles, CA, USA.

J Gen Intern Med 34(10): 1968-70

DOI: $10.1007 / \mathrm{s} 11606-019-05072-\mathrm{w}$

(C) Society of General Internal Medicine 2019

\section{INTRODUCTION}

Emergency room (ER) overcrowding occurs frequently and can result in poor clinical outcomes. Active bed management by hospitalists has been shown to improve ER patient flow without negative impacts on clinical outcomes. ${ }^{1}$ Studies from Korea have shown transfers to regional hospitals can reduce ER lengths of stay (LOS) for patients awaiting admission, though by an average of less than $2 \mathrm{~h} .{ }^{2,3}$ Despite these modest reductions in ER LOS, transfers directed by hospitalists may help alleviate ER overcrowding.

Our hospital system began a hospitalist-directed transfer process to reduce ER overcrowding in 2011 by developing a process for intra-health system transfers. As part of the process, hospitalists at a referral center ER screen patients based on clinical judgment for transfer and directly admit consenting patients to an affiliated hospital with greater bed availability. To assess the impact and safety of this hospitalist-led intervention to reduce ER LOS, and thereby alleviate overcrowding, we examined the differences in ER LOS and hospital outcomes between patients transferred and those who qualified for transfer but declined.

\section{METHODS}

Between August 2013 and June 2015, 1016 admitted patients met the criteria for transfer based on our hospitalists' assessment of stability and care requirements. Of these, 515 successfully transferred while 501 remained, and we compared outcomes between these groups. Five investigators conducted chart abstraction using a standardized key. Data gathered included demographics, the Elixhauser score (a comorbidity index predictive of LOS and in-hospital death), mode of arrival, vital signs, significant events during hospitalization, ER LOS (in hours roomed in ER), hospital LOS (in days), discharge disposition, and 30-day readmission rates.

Statistical analysis included univariate analysis, propensity scoring, and multiple multivariate regression models on the weighted cohort based on patient's transfer status. To address sampling bias, we estimated a propensity score for each subject using variables that were significantly different in the univariate analysis. In this weighted cohort $(n=1011)$, vital signs, significant events, and the Elixhauser scores were similar between the two cohorts.

\section{RESULTS}

On univariate analysis, transferred patients were slightly younger in age (61.3 vs. 64.6; $p$ 0.01). Those who remained at the

Table 1 Patient Characteristics and Transfer Outcomes

\begin{tabular}{|c|c|c|c|c|}
\hline & $\begin{array}{l}\text { Overall } \\
(n=1016)\end{array}$ & $\begin{array}{l}\text { Admitted to reference } \\
\text { hospital }(n=501)\end{array}$ & $\begin{array}{l}\text { Transferred to affiliated } \\
\text { hospital }(n=515)\end{array}$ & $p$ value \\
\hline Age (SD) & $62.9(20.6)$ & $64.6(20.6)$ & $61.3(20.5)$ & 0.01 \\
\hline Gender (female) & $531(52.2 \%)$ & $250(49.9 \%)$ & $281(54.5 \%)$ & 0.14 \\
\hline Elixhauser score & $6.1(6.6)$ & $7.77(6.8)$ & $4.47(6.0)$ & $<0.001$ \\
\hline Hospitalized in prior 30 days & $127(12.5 \%)$ & $78(15.5 \%)$ & $49(9.51 \%)$ & 0.003 \\
\hline Vital signs & & & & $<0.001$ \\
\hline Normal & $505(49.9 \%)$ & $211(42.37 \%)$ & $294(57.2 \%)$ & \\
\hline 1 abnormal & $351(34.7 \%)$ & $201(40.3 \%)$ & $150(29.2 \%)$ & \\
\hline$>1$ abnormal & $156(15.4 \%)$ & $86(17.2 \%)$ & $70(13.46 \%)$ & \\
\hline ER LOS & $13.0(8.6)$ & $16.9(10.3)$ & $9.2(4.0)$ & $<0.001$ \\
\hline \multicolumn{5}{|l|}{ Significant events } \\
\hline None & $960(94.4 \%)$ & $465(92.8 \%)$ & $495(96.1 \%)$ & 0.02 \\
\hline Upgrade to ICU & $36(3.44 \%)$ & $24(4.79 \%)$ & $11(2.14 \%)$ & 0.02 \\
\hline Code or rapid response & $4(0.39 \%)$ & $3(0.60 \%)$ & $1(0.19 \%)$ & 0.37 \\
\hline Hospital LOS & $5.93(6.63)$ & $5.92(7.17)$ & $5.94(6.07)$ & 0.95 \\
\hline Readmission in 30 days & $201(19.8 \%)$ & $116(23.2 \%)$ & $85(16.5 \%)$ & 0.007 \\
\hline
\end{tabular}

Published online June 3, 2019 
reference hospital were more likely to have higher Elixhauser scores, abnormal vital signs, and to be upgraded to the ICU (Table 1). Transferred patients had shorter ER stay (9.23 vs. $16.94 \mathrm{~h} ; p<0.001)$ and lower rates of readmission within 30 days of the index hospitalization (23.29\% vs. $16.57 \%$; $p$ 0.007). There was no significant difference in hospital LOS, discharge disposition, or insurance status between the two groups.

Our multivariate regression analyses employed the aforementioned propensity-matched cohort (Table 2). In the hospital LOS analysis, recent hospitalization, the Elixhauser scores, and significant events were significant predictors of hospital LOS. Transfer status was not a significant predictor of hospital LOS nor of an adverse event. In the ER LOS analysis, transfer status was a significant predictor of ER LOS, being shorter by $7.5 \mathrm{~h}$ $(p<0.001)$. The Elixhauser score, vital signs, and recent hospitalization were not significant predictors of ER LOS in this model.

Finally, in examining the 30-day readmission rates following the index hospitalization, transfer status was not a significant predictor in the propensity-matched analysis. The Elixhauser scores, being discharged from the hospital in the prior 30 days to the index admission, and having more than 1 abnormal vital sign on ER presentation, were significant covariates. Additionally, of those who were readmitted in 30 days, in the univariate analysis, $52.94 \%$ of transfers to the affiliated hospital during the index hospitalization returned to the affiliated hospital ER while $82.9 \%$ of those who declined transfer returned to the reference hospital $(p<0.001)$.

\section{DISCUSSION}

This study showed that hospitalist-directed transfer and direct admission of stable ER patients has the potential to shorten ER LOS. In our program, these transfers were not associated with higher rates of significant events, longer hospital LOS, or 30day readmission rates compared with patients remaining at the reference hospital. Based on our finding in the multivariable regression analysis that transferring patients reduced the ER LOS by an average of $7.5 \mathrm{~h}$, this program saves our 41-bed ER that services 50,000 patients annually approximately $1936 \mathrm{~h}$ per year in LOS. Further research into the economic, patient satisfaction, and quality of care implications of this intervention is warranted.

Corresponding Author: Roswell Guinn, M.D., Ph.D; Hospitalist Division of the Department of Medicine, Hospitalist Service VA Greater Los Angeles Healthcare System, 11301 Wilshire Blvd, B500, Room 3238 (Mail code: 111), Los Angeles, CA 90073, USA (e-mail: roswellquinn@mednet.ucla.edu).

\section{Compliance with Ethical Standards:}

Conflict of Interest: The authors declare that they do not have a conflict of interest. 


\section{REFERENCES}

1. Howell E, Bessman E, Kravet S, Kolodner K, Marshall R, Wright S. Active Bed Management by Hospitalists and Emergency Department Throughput. Ann Intern Med 2008; 149:804-810.

2. Ro, Y. S., Shin, S. D., Song, K. J., Cha, w. C., Cho, J. S. Triage-based resource allocation and clinical treatment protocol on outcome and length of stay in the emergency department. Emerg Med Australas 2015;27:328335 .
3. Cha, w. C., Shin, S. D., Song, K. J., Jung, S. K., Suh, G. J. Effect of an Independent-capacity Protocol on Overcrowding in an Urban Emergency Department. Acad Emerg Med 2009;16:1277-1283.

Publisher's Note Springer Nature remains neutral with regard to jurisdictional claims in published maps and institutional affiliations. 